\title{
Different risks of nodal metastasis by tumor location in remnant gastric cancer after curative gastrectomy for gastric cancer
}

\author{
Kazuya Takahashi ${ }^{1} \cdot$ Takaki Yoshikawa $^{1} \oplus \cdot$ Shinji Morita $^{1} \cdot$ Takahiro Kinoshita $^{2} \cdot$ Masahiro Yura $^{1} \cdot$ Sho Otsuki $^{1}$. \\ Masanori Tokunaga ${ }^{2} \cdot$ Yukinori Yamagata $^{1} \cdot$ Akio Kaito $^{2} \cdot$ Hitoshi Katai $^{1}$
}

Received: 23 April 2019 / Accepted: 4 July 2019 / Published online: 13 July 2019

(c) The International Gastric Cancer Association and The Japanese Gastric Cancer Association 2019

\begin{abstract}
Background Curative surgery for remnant gastric cancer (RGC) after gastrectomy for gastric cancer (GC) can be challenging. We examined the risk factors for lymph node metastasis in RGC, especially for tumors located at the greater curvature (G) or non-greater curvature (NG), to determine the appropriate indications of curative surgery.

Methods Data from the two high-volume centers of Japan between 1998 and 2018 were retrospectively reviewed. Among the 137 patients enrolled in this study, 34 were classified as the G group and 103 as the NG group. The incidence of lymph node metastasis and its risk factors was evaluated.

Results Lymph node metastasis was observed in 21.2\% (29/137), including 38.2\% (13/34) in the G group and 15.5\% (16/103) in the NG group $(p=0.008)$. A logistic regression analysis showed that tumor location of $\mathrm{G}$ or NG $(p=0.042)$, tumor size $(p=0.002)$ and depth of invasion $(p=0.009)$ were significant independent risk factors for nodal metastasis. Risk classification using these factors showed that clinical T1-T2 with a maximum size $<35 \mathrm{~mm}$ located at the non-greater curvature had the lowest nodal metastatic risk (4.3\%).

Conclusions Tumor location at the G or NG was a significant risk factor for nodal metastasis in RGC. When selecting curative surgery for RGC, physicians should consider the nodal metastatic risk calculated by the tumor location, size and depth of invasion.
\end{abstract}

Keywords Remnant gastric cancer · Nodal metastasis · Initial gastrectomy for gastric cancer

\section{Introduction}

Remnant gastric cancer (RGC), defined as gastric cancer (GC) that develops at the remnant stomach after gastrectomy for benign or malignant disease, is relatively rare. Since gastrectomy is now inapplicable for peptic ulcer, the major population of RGC patients has been RGC after gastrectomy for GC. However, while complete tumor removal is essential for curing RGC, curative surgery for RGC can be difficult, especially when the primary surgery was curative lymphadenectomy for GC.

Takaki Yoshikawa tayoshik@ncc.go.jp

1 Gastric Surgery Division, National Cancer Center Hospital, 5-1-1 Tsukiji, Chuo-ku, Tokyo 104-0045, Japan

2 Gastric Surgery Division, National Cancer Center Hospital East, 6-5-1 Kashiwanoha, Kashiwa, Chiba 277-8577, Japan
The first issue with RGC is the technical difficulty of associated surgery. Adhesion around the remnant stomach is severe, which may easily cause massive bleeding or injury to the organs around the stomach [1]. The second issue is the need for splenectomy. RGC is mostly limited to the upper third of the stomach. As the nodal station of the lesser curvature side is completely dissected at the primary surgery, all lymphatic flow from the remnant stomach reportedly travels to the greater curvature $(\mathrm{G})$, regardless of the location [2]. Therefore, total gastrectomy with splenectomy is recommended for RGC [3]. The third issue is aging. Since the mean interval after operations for GC is approximately 10 years [4-7], most patients with RGC are elderly, a population that often has several co-morbidities. Given these problems, surgery for RGC may result in high morbidity and mortality [8]. The decision to perform surgery must, therefore, be made after considering the morbidities and nodal metastatic risk; however, the metastatic risk for RGC has not been fully evaluated. 
For primary GC, whether the location is at the G or not (NG) is a recent topic of interest for determining the extent of nodal dissection. A JCOG0110 phase III study targeting primary GC not invading the G clearly showed that metastatic rate to the nodes along the splenic artery or the splenic hilar nodes was very low [9]. In contrast, RGC located at the NG has shown a lymphatic flow to the G side [2]. However, whether or not these tumors show nodal metastasis equally to the RGC located at the $\mathrm{G}$ is unclear at present.

To clarify these points, we explored the risk factors for lymph node metastasis in patients with RGC, with close consideration of the tumor location at the G or NG. We also tried to calculate the risk stratified by significant risk factors, to which physicians could refer when determining the appropriate indication of lymphadenectomy for RGC.

\section{Materials and methods}

\section{Patient}

The study was conducted as a subgroup analysis of the "remnant gastric cancer project of the National Cancer Center, Japan". The main purpose of this project is to clarify whether splenectomy is avoidable for advanced remnant gastric cancer. The main result of this project was reported previously [10]. This project retrospectively collected the data of patients who had received gastrectomy before, developed gastric cancer at the remnant stomach, and received second gastrectomy at the National Cancer Center Hospital $(\mathrm{NCCH})$ and the National Cancer Center Hospital East (NCCHE) between 1998 and 2018.

Among the 290 patients enrolled in this project, the present study selected the patients who met the following criteria: (1) RGC developed after distal or pylorus-preserving gastrectomy for GC and (2) underwent total gastrectomy for RGC located at the upper third of the stomach. Principally, \#2 and \#4sa were dissected for early disease. In addition, \#10 and \#11 were dissected for advanced disease and in case that those nodes were suspected to have metastasis even for early tumors. When \#4sb was not completely dissected at the initial operation, \#4sb was added. When initial surgery was pylorus-preserving gastrectomy, \#5 was also dissected. These strategy for nodal dissection was determined not depending on the tumor location.

\section{Data collection}

The "remnant gastric cancer project" collected data on the following patient characteristics: gender, age, initial gastrectomy, interval between the initial gastrectomy and total gastrectomy for RGC, macroscopic type, tumor size, depth of invasion, histological type, liver metastasis, intraoperative peritoneal lavage cytology, resection of adjacent organs for RGC, number of lymph nodes, nodal status and lymph node stations. By reviewing the picture of the resected tumor, the patients were classified into two groups: the G group (those with tumors invading the greater curvature line), and the NG group (all other patients). The clinicopathological factors were recorded according to the 15 th edition of the Japanese Classification of Gastric Carcinoma [9].

\section{Statistical analyses}

The incidence of nodal metastasis and its order were compared between the $\mathrm{G}$ and NG groups. Comparisons between the two groups were analyzed by the Mann-Whitney $U$ test, Kruskal-Wallis test or using the Chi square method. A logistic regression analysis was used to evaluate the risk factors for lymph node metastasis. A $p$ value $<0.05$ was considered significant. All statistical analyses were performed using The IBM SPSS Statistics 22.0 (IBM, Corp., Armonk, NY, USA).

\section{Results}

\section{Patient characteristics}

Figure 1 shows a flow diagram of the present study. Among 290 patients enrolled in this "remnant gastric cancer project", 137 were entered into this study. Thirty-four patients were classified into the G group and 103 into the NG group.

Table 1 shows the background clinicopathological characteristics. Distal gastrectomy was the most common procedure as the initial surgery, performed in 121 patients $(88.3 \%)$. There was no significant difference in the reconstruction method between the two groups. The interval between initial and total gastrectomy for RGC was significantly longer in the $\mathrm{G}$ group than in the NG group $(p=0.008)$. In the NG group, the depth of invasion was deeper, undifferentiated histology more frequent and nodal metastasis more apparent than in the $\mathrm{G}$ group.

Table 2 shows proportion of each nodal dissection of the both groups. When stratified by the tumor depth, nodal dissection rate was almost similar between the $\mathrm{G}$ and the NG except \#2 for cT2-4.

\section{Incidence of lymph node metastasis}

A total of 29 patients had lymph node metastasis $(21.2 \%)$, which was significantly more frequent in the G group (13/34, $38.2 \%)$ than in the NG group $(16 / 103,15.5 \%)(p=0.008)$. The metastatic incidence to each nodal station in the $\mathrm{G}$ group was $>15 \%$, while that in the NG group was $<10 \%$ (Table 3). The most frequent nodal station of the G group 


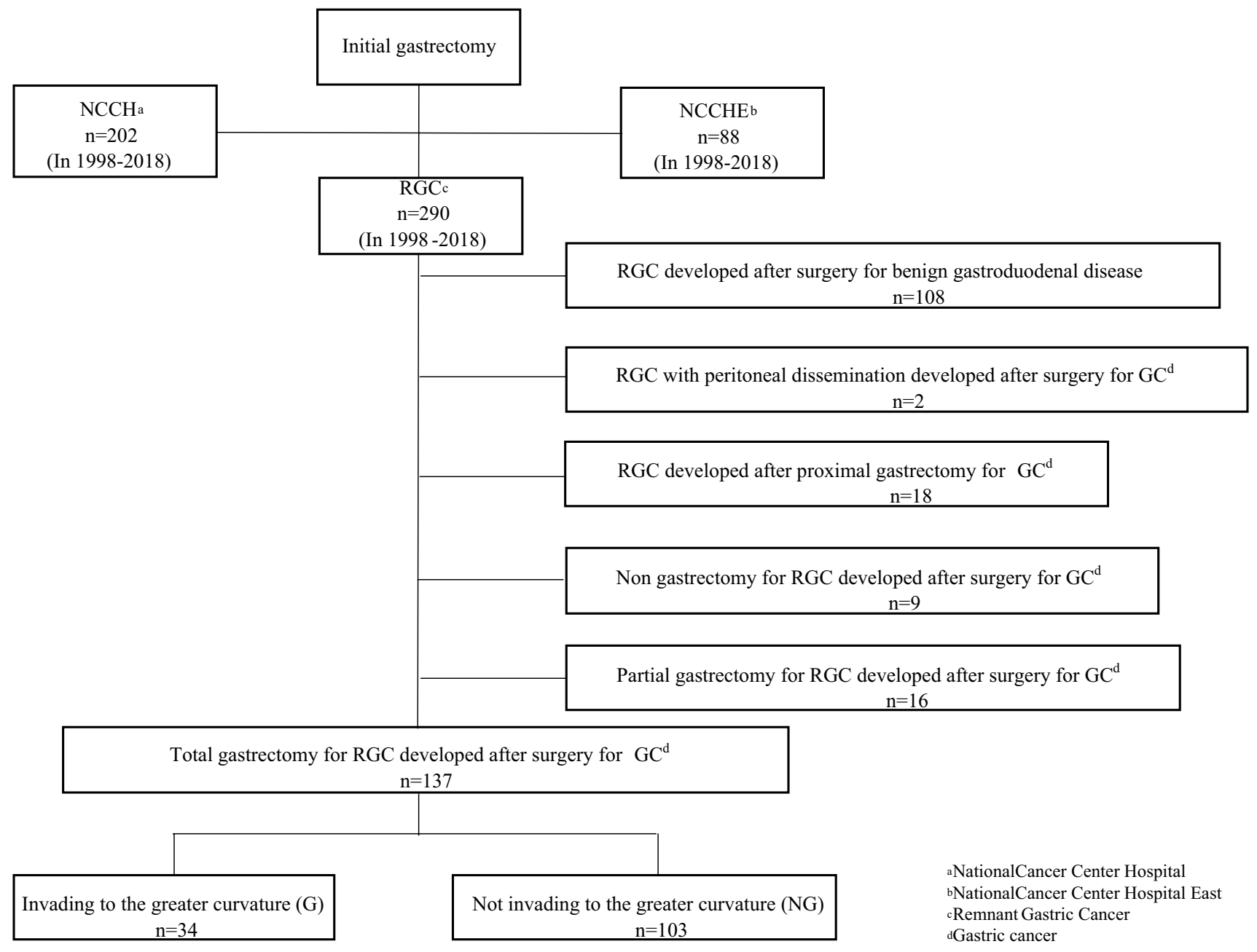

Fig. 1 Patient flow diagram

was \#4sb and \#10 followed by \#4sa and \#2, while that of the NG group was \#10 followed by \#2, \#4sa and \#4sb.

\section{Risk factors for lymph node metastasis}

The univariate analysis showed that the tumor location $(p=0.006)$, histological type $(p=0.020)$, tumor size $(p<0.001)$ and depth of invasion $(p<0.001)$ were significant risk factors for nodal metastasis. According to the multivariate analysis, tumor location, tumor size and depth of invasion were identified as significant independent risk factors for lymph node metastasis (Table 4).

\section{Incidence of lymph node metastasis stratified by the significant risk factors}

Based on the significant risk factors, the nodal metastatic risk was stratified into eight groups. cT1-T2 tumors located at the $\mathrm{NG}<35 \mathrm{~mm}$ in diameter showed a low incidence of lymph node metastasis, whereas cT3-T4 tumors located at the $\mathrm{G} \geq 35 \mathrm{~mm}$ in diameter showed a high incidence (Table 5).

\section{Discussion}

In the present study, we explored the risk factors for lymph node metastasis in patients with RGC, with particular focus on the tumor location at the G or NG. We then tried to determine the risk stratified by significant risk factors. Tumor location was a significant risk factor for nodal metastasis in RGC. Furthermore, the nodal risk was able to be stratified by tumor location, size and depth. When selecting curative surgery for RGC, physicians should consider the nodal metastatic risk calculated by these factors.

The major finding of this study is that the risk of lymph node metastasis was totally different depending on the location of RGC. The lymph node metastatic rate was 
Table 1 Background characteristics

\begin{tabular}{|c|c|c|c|c|}
\hline Factor & Number of patients $(n=137)$ & $\mathrm{G}(n=34)$ & NG $(n=103)$ & $p$ value \\
\hline Gender & & & & 0.118 \\
\hline Male & $100(73.0 \%)$ & $21(61.7 \%)$ & $79(76.7 \%)$ & \\
\hline Female & $37(27.0 \%)$ & $13(38.3 \%)$ & $24(23.3 \%)$ & \\
\hline Age (years) (median, range) & $68 \pm 9.28(30-85)$ & $70 \pm 8.50$ & $67 \pm 9.45$ & 0.102 \\
\hline Initial gastrectomy & & & & 0.392 \\
\hline Billroth I & $83(60.6 \%)$ & $20(58.8 \%)$ & $63(61.2 \%)$ & \\
\hline Billroth II & $25(18.2 \%)$ & $9(26.5 \%)$ & $16(15.5 \%)$ & \\
\hline Roux-en-Y & $13(9.5 \%)$ & $3(8.8 \%)$ & $10(9.7 \%)$ & \\
\hline Pylorus-preserving-gastrectomy & $16(11.7 \%)$ & $2(5.9 \%)$ & $14(13.6 \%)$ & \\
\hline Interval (years) (median, range) & $8 \pm 10.4(2$ months -51$)$ & $10.6 \pm 14.6$ & $7 \pm 7.86$ & 0.008 \\
\hline Macroscopic type & & & & 0.835 \\
\hline сType0 & $87(63.5 \%)$ & $19(55.9 \%)$ & $68(66.0 \%)$ & \\
\hline cType1 & $8(5.8 \%)$ & $3(8.8 \%)$ & $5(4.9 \%)$ & \\
\hline cType2 & $15(10.9 \%)$ & $5(14.7 \%)$ & $10(9.7 \%)$ & \\
\hline cType3 & $13(9.5 \%)$ & $3(8.8 \%)$ & $10(9.7 \%)$ & \\
\hline cType4 & $9(6.6 \%)$ & $3(8.8 \%)$ & $6(5.8 \%)$ & \\
\hline cType5 & $5(3.7 \%)$ & $1(3.0 \%)$ & $4(3.9 \%)$ & \\
\hline Tumor size (mm) (median, range) & $35 \pm 36.8(1-204)$ & $48 \pm 47.5$ & $35 \pm 31.9$ & 0.115 \\
\hline Depth of invasion & & & & 0.004 \\
\hline $\mathrm{cT} 1$ & $87(63.5 \%)$ & $16(47.1 \%)$ & $71(68.9 \%)$ & \\
\hline $\mathrm{cT} 2$ & $18(13.2 \%)$ & $6(17.6 \%)$ & $12(11.7 \%)$ & \\
\hline cT3 & $8(5.8 \%)$ & 0 & $8(7.8 \%)$ & \\
\hline $\mathrm{cT} 4 \mathrm{a}$ & $19(13.8 \%)$ & $9(26.5 \%)$ & $10(9.7 \%)$ & \\
\hline $\mathrm{cT} 4 \mathrm{~b}$ & $5(3.7 \%)$ & $3(8.8 \%)$ & $2(1.9 \%)$ & \\
\hline Histological type & & & & 0.009 \\
\hline Differentiated & $60(43.8 \%)$ & $8(23.5 \%)$ & $52(50.5 \%)$ & \\
\hline Undifferentiated & $77(56.2 \%)$ & $26(76.5 \%)$ & $51(49.5 \%)$ & \\
\hline Liver metastasis & & & & $\mathrm{N}$ \\
\hline $\mathrm{HO}$ & $137(100 \%)$ & $34(100 \%)$ & $103(100 \%)$ & \\
\hline $\mathrm{H} 1$ & 0 & 0 & 0 & \\
\hline Intraoperative peritoneal lavage cytology & & & & 0.054 \\
\hline CY0 & $92(67.2 \%)$ & $26(76.5 \%)$ & $66(64.1 \%)$ & \\
\hline CY1 & $3(2.2 \%)$ & $2(5.9 \%)$ & $1(0.9 \%)$ & \\
\hline CYX & $42(30.6 \%)$ & $6(17.6 \%)$ & $36(35.0 \%)$ & \\
\hline Resection of adjacent organs & & & & 0.05 \\
\hline No & $69(50.4 \%)$ & $12(35.3 \%)$ & $57(55.3 \%)$ & \\
\hline Yes & $68(49.6 \%)$ & $22(64.7 \%)$ & $46(44.7 \%)$ & \\
\hline Number of lymph node (median, range) & $12 \pm 11.3(1-66)$ & $14 \pm 13.9$ & $10 \pm 10.1$ & 0.092 \\
\hline Lymph node metastasis & & & & 0.035 \\
\hline pNO & $108(78.8 \%)$ & $21(61.7 \%)$ & $87(84.5 \%)$ & \\
\hline $\mathrm{pN} 1$ & $10(7.3 \%)$ & $4(11.8 \%)$ & $6(5.8 \%)$ & \\
\hline $\mathrm{pN} 2$ & $12(8.8 \%)$ & $5(14.7 \%)$ & $7(6.8 \%)$ & \\
\hline $\mathrm{pN} 3$ & $7(5.1 \%)$ & $4(11.8 \%)$ & $3(2.9 \%)$ & \\
\hline
\end{tabular}

around 2.8 times greater in the $\mathrm{G}$ group than in the $\mathrm{NG}$ group. In primary proximal advanced GC, the nodal metastatic risk was reportedly $53-77 \%$ in the $\mathrm{G}$ and $42-76 \%$ in the NG in retrospective analyses, while it was $57.8 \%$ in the latter in the JCOG0110 phase III study [11-14]. Thus, the nodal metastatic risk of the primary disease seems to be similar regardless of location, which is a totally different finding from RGC. However, several studies have also shown that NG tumors have a very low potential to metastasize to the nodes along the $G[12,14]$. Such a metastatic 
Table 2 The proportion of each nodal dissection in the G and NG group

\begin{tabular}{|c|c|c|c|c|c|c|}
\hline \multirow{2}{*}{$\begin{array}{l}\text { Lymph node } \\
\text { station }\end{array}$} & \multicolumn{3}{|l|}{ G } & \multicolumn{3}{|l|}{ NG } \\
\hline & All & cT1 & cT2-4 & All & cT1 & cT2-4 \\
\hline \#2 & $31 / 34(91.2 \%)$ & $14 / 16(87.5 \%)$ & $17 / 18(94.4 \%)$ & $88 / 103(85.4 \%)$ & $64 / 71(90.1 \%)$ & $24 / 32(75.0 \%)$ \\
\hline$\# 4$ sa & $30 / 34(88.2 \%)$ & $15 / 16(93.8 \%)$ & $15 / 18(83.3 \%)$ & $91 / 103(88.3 \%)$ & $62 / 71(87.3 \%)$ & $29 / 32(90.6 \%)$ \\
\hline$\# 4 \mathrm{sb}$ & $14 / 34(41.2 \%)$ & $7 / 16(43.8 \%)$ & $7 / 18(38.9 \%)$ & $49 / 103(47.6 \%)$ & $36 / 71(50.7 \%)$ & $13 / 32(40.6 \%)$ \\
\hline$\# 10$ & $20 / 34(58.8 \%)$ & $5 / 16(31.3 \%)$ & $15 / 18(83.3 \%)$ & $45 / 103(43.7 \%)$ & $19 / 71(26.8 \%)$ & $26 / 32(81.3 \%)$ \\
\hline
\end{tabular}

Table 3 Incidence of lymph node metastasis and $\mathrm{G}$ vs. NG group assignment

\begin{tabular}{lrr}
\hline $\begin{array}{l}\text { Lymph node } \\
\text { station }\end{array}$ & \multicolumn{2}{l}{ NG } \\
\hline all & $13 / 34(38.2 \%, 95 \%$ CI $0.248-0.757)$ & $16 / 103(15.5 \%, 95 \%$ CI $1.038-2.053) 0.008$ \\
$\# 2$ & $5 / 31(16.1 \%, 95 \%$ CI $0.276-1.233)$ & $7 / 88(8.0 \%, 95 \%$ CI $0.795-2.118) 0.295$ \\
$\# 4 \mathrm{sa}$ & $5 / 30(16.7 \%, 95 \%$ CI $0.222-0.916)$ & $5 / 91(5.5 \%, 95 \%$ CI $0.827-2.903) 0.118$ \\
$\# 4 \mathrm{sb}$ & $3 / 14(21.4 \%, 95 \%$ CI $0.130-0.771)$ & $2 / 49(4.1 \%, 95 \%$ CI $0.687-5.970) 0.068$ \\
$\# 10$ & $4 / 20(20.0 \%, 95 \%$ CI $0.250-1.259)$ & $4 / 45(8.9 \%, 95 \%$ CI $0.706-2.931) 0.238$ \\
\hline
\end{tabular}

Table 4 Risk factors for lymph node metastasis by the logistic regression analysis

Table 5 Incidence of lymph node metastasis stratified by the significant risk factors

\begin{tabular}{|c|c|c|c|c|c|c|c|}
\hline \multirow[t]{2}{*}{ Variable } & \multirow[t]{2}{*}{ Total } & \multicolumn{2}{|c|}{ Lymph node metastasis } & \multirow{2}{*}{$\begin{array}{l}\text { Univariate } \\
p \text { value }\end{array}$} & \multicolumn{3}{|c|}{ Multivariate } \\
\hline & & No & Yes & & OR $(B)$ & OR $(95 \% \mathrm{CI})$ & $p$ value \\
\hline Tumor location & & & & 0.006 & 2.793 & $1.040-7.500$ & 0.042 \\
\hline NG & 103 & $87(84.5 \%)$ & $16(15.5 \%)$ & & & & \\
\hline $\mathrm{G}$ & 34 & $21(61.8 \%)$ & $13(38.2 \%)$ & & & & \\
\hline Histological type & & & & 0.020 & 1.918 & $0.676-5.441$ & 0.221 \\
\hline Differentiated & 60 & $53(88.3 \%)$ & $7(11.7 \%)$ & & & & \\
\hline Undifferentiated & 77 & $55(71.4 \%)$ & $22(28.6 \%)$ & & & & \\
\hline Tumor size & & & & $<0.001$ & 4.271 & $1.253-14.560$ & 0.002 \\
\hline$<35$ & 62 & $58(93.5 \%)$ & $4(6.5 \%)$ & & & & \\
\hline$\geq 35$ & 75 & $50(66.7 \%)$ & $25(33.3 \%)$ & & & & \\
\hline Depth of invasion & & & & $<0.001$ & 3.846 & $1.395-10.702$ & 0.009 \\
\hline $\mathrm{cT} 1-\mathrm{T} 2$ & 106 & $93(87.7 \%)$ & $13(12.3 \%)$ & & & & \\
\hline cT3-T4 & 31 & $15(48.4 \%)$ & $16(51.6 \%)$ & & & & \\
\hline Macroscopic type & & & & 0.363 & 0.983 & $0.189-5.120$ & 0.984 \\
\hline Non-type 4 & 128 & $102(79.7 \%)$ & $26(20.3 \%)$ & & & & \\
\hline Type 4 & 9 & $6(66.7 \%)$ & $3(33.3 \%)$ & & & & \\
\hline
\end{tabular}

\begin{tabular}{llllll}
\hline Case & Variable & \multicolumn{2}{c}{} & $\begin{array}{l}\text { Incidence of lymph } \\
\text { node metastasis }\end{array}$ & 95\% CI \\
\cline { 2 - 4 } & Tumor location & Tumor size & Depth of invasion & & \\
\hline 1 & $\mathrm{NG}$ & $<35$ & $\mathrm{cT} 1-\mathrm{T} 2$ & $2 / 47(4.3 \%)$ & $0.00519-0.145$ \\
2 & $\mathrm{NG}$ & $<35$ & $\mathrm{cT} 3-\mathrm{T} 4$ & $\mathrm{~N}$ & $\mathrm{~N}$ \\
3 & $\mathrm{NG}$ & $\geq 35$ & $\mathrm{cT} 1-\mathrm{T} 2$ & $6 / 37(16.2 \%)$ & $0.0619-0.320$ \\
4 & $\mathrm{NG}$ & $\geq 35$ & $\mathrm{cT} 3-\mathrm{T} 4$ & $8 / 17(47.1 \%)$ & $0.229-0.722$ \\
5 & $\mathrm{G}$ & $<35$ & $\mathrm{cT} 1-\mathrm{T} 2$ & $2 / 13(15.4 \%)$ & $0.0192-0.454$ \\
6 & $\mathrm{G}$ & $<35$ & $\mathrm{cT} 3-\mathrm{T} 4$ & $\mathrm{~N}$ & $\mathrm{~N}$ \\
7 & $\mathrm{G}$ & $\geq 35$ & $\mathrm{cT} 1-\mathrm{T} 2$ & $3 / 9(33.3 \%)$ & $0.0749-0.701$ \\
8 & $\mathrm{G}$ & $\geq 35$ & $\mathrm{cT} 3-\mathrm{T} 4$ & $8 / 12(66.7 \%)$ & $0.349-0.901$ \\
\hline
\end{tabular}


tendency is expected to be preserved even in RGC at the $\mathrm{NG}$, in which all lymphatic flow from the remnant stomach goes to the $\mathrm{G}$ [2].

The present study clearly demonstrated that the independent risk factors for nodal metastasis in the RGC were the tumor location, size and depth. We were also able to stratify RGC into several subgroups by combining these risk factors. In particular, we identified the subgroup with a very low nodal metastatic risk: patients with clinical T1-T2 with a maximum size $<35 \mathrm{~mm}$ located at the NG. In this subgroup, the metastatic risk was $<5 \%$, suggesting that physicians may avoid radical surgery if the surgical mortality is estimated to exceed $5 \%$. Since surgery for RGC is associated with issues such as adhesion, the need for splenectomy and co-morbidities accompanied by aging, the surgical risk would easily exceed 5\%. Local resection may be a viable treatment choice for this subset.

In this study, the tumor size and clinical tumor depth were both independent risk factors for lymph node metastasis, which is not surprising. Tumor size was reportedly a risk factor for nodal metastasis in primary GC, especially early GC $[15,16]$. Tumor depth is also closely associated with nodal metastasis in primary disease [3, 17].

Several limitations associated with the present study warrant mention. The first issue is the consistency of the preoperative staging. The present study included old cases treated before 2000 because RGC is a rare disease. Over this relatively long study period, the modality for the diagnosis improved. Second, difference of nodal dissection rate could affect the present results. Although that was almost similar between the $\mathrm{G}$ and the NG, dissection rate for \#2 in cT2-4 was higher in the G than the NG. Higher dissection rate would cause higher metastatic rate. However, \#2 nodal station is always preserved in the remnant gastric cancer of this cohort and must be dissected during the surgery. Thus, difference of \#2 dissection rate must be due to harvesting. On the other hand, the most different incidence of nodal metastasis between the G and NG was \#4sb considering the $p$ value. However, proportion of nodal dissection for \#4sb was greater in the NG than the G. Thus, metastatic rate at \#4sb was more underestimated in the $\mathrm{G}$ than the NG. It would be unlikely that the difference of nodal dissection affected the present results. Third, we did not refer to the survival in this study. Ideally, whether or not lymph nodes should be dissected would be evaluated based on the therapeutic index, which is calculated by multiplying the metastatic rate and 5-year survival rate. However, the follow-up period was limited, especially in the patients who had been treated within the past couple of years. In addition, the optimal adjuvant chemotherapy regimen changed over the relatively lengthy study period. We, therefore, considered the reliability of the survival data to be limited.
In conclusion, tumor location at the $\mathrm{G}$ or NG was a significant risk factor for nodal metastasis in RGC after initial gastrectomy for GC. We were also able to stratify the risk of nodal metastasis by the tumor location, size and depth of invasion. Based on these risk factors, the physicians should determine the indication of surgery for RGC after initial gastrectomy for GC.

\section{Compliance with ethical standards}

Conflict of interest The authors declare that they have no conflicts of interest.

Ethical approval All the procedures followed were in accordance with the ethical standards of the responsible committee on human experimentation (institutional and national) and with the 1964 Declaration of Helsinki and later versions. Informed consent to be included in the study, or the equivalent, was obtained from all patients.

\section{References}

1. Kwon IG, Cho I, Choi YY, Hyung WJ, Kim CB, Noh SH. Risk factors for complications during surgical treatment of remnant gastric cancer. Gastric Cancer. 2015;18:390-6.

2. Honda S, Bando E, Makuuchi R, Tokunaga M, Tanizawa Y, Terashima M, et al. Effects of initial disease status on lymph flow following gastrectomy in case of carcinoma in the remnant stomach. Gastric Cancer. 2017;20:457-64.

3. Ohashi M, Morita S, Fukagawa T, Kushima R, Katai H. Surgical treatment of non-early gastric remnant carcinoma developing after distal gastrectomy for gastric cancer. J Surg Oncol. 2015;111:208-12.

4. Kodera Y, Yamaura Y, Torii A, Uesaka K, Hirai T, Kito T, et al. Gastric remnant carcinoma after partial gastrectomy for benign and malignant gastric lesions. J Am Coll Surg. 1996;182:1-6.

5. Ohashi M, Katai H, Fukagawa T, Gotoda T, Sano T, Sasako M. Cancer of the gastric stump following distal gastrectomy for cancer. B J Surg. 2007;94:92-5.

6. Kunisaki C, Shimada H, Nomura M, Hosaka N, Akiyama H, Yamaoka H, et al. Lymph node dissection in surgical treatment for remnant stomach cancer. Hepatogastroenterology. 2002;49:580-4.

7. Sinning C, Schaefer N, Standop J, Hirner A, Wolff M. Gastric stump carcinoma-epidemiology and current concepts in pathogenesis and treatment. Eur J Surg Oncol. 2007;33:133-9.

8. Ahn HS, Kim JW, Yoo MW, Park DJ, Lee HJ, Yang HK, et al. Clinicopathological features and surgical outcomes of patients with remnant gastric cancer after a distal gastrectomy. Ann Surg Oncol. 2008;15(6):1632-9.

9. Japanese Gastric Cancer Association. Japanese classification of gastric carcinoma. 15th ed. Tokyo: Kanehara Publisher; 2017.

10. Watanabe M, Kinoshita T, Morita S, Tokunaga M, Yoshikawa $\mathrm{T}$, Katai H, et al. Clinical impact of splenic hilar dissection with splenectomy for gastric stump cancer. Eur J Surg Oncol. 2019. https://doi.org/10.1016/j.ejso.2019.03.030.

11. Sano T, Sasako M, Mizusawa J, Yamamoto S, Katai H, Fujitani $\mathrm{K}$, et al. Randomized controlled trial to evaluate splenectomy in total gastrectomy for proximal gastric carcinoma. Ann Surg. 2017;265:277-83.

12. Watanabe M, Kinoshita T, Enomoto N, Shibasaki H, Nishida T. Clinical significance of splenic hilar dissection with splenectomy 
in advanced proximal gastric cancer: an analysis at a single institution in Japan. World J Surg. 2016;40:1165-71.

13. Maezawa Y, Aoyama T, Yamada T, Kano K, Hayashi T, Yoshikawa $\mathrm{T}$, et al. Priority of lymph node dissection for proximal gastric cancer invading the greater curvature. Gastric Cancer. 2018;21:569-72.

14. Yura M, Yoshikawa T, Otsuki S, Yamagata Y, Morita S, Nishida $\mathrm{T}$, et al. The therapeutic survival benefit of splenic hilar nodal dissection for advanced proximal gastric cancer invading the greater curvature. Ann Surg Oncol. 2019;26:829-35.

15. Hatta W, Gotoda T, Oyama T, Kawata N, Takahashi A, Shimosegawa $\mathrm{T}$, et al. A scoring system to stratify curability after endoscopic submucosal dissection for early gastric cancer: " $\mathrm{eCu}-$ rasystem". Am J Gastroenterol. 2017;112:874-81.
16. Newman E, Brennan MF, Hochwald SN, Harrison LE, Karpeh MS Jr. Gastric remnant carcinoma: just another proximal gastric cancer or a unique entity? Am J Surg. 1997;173:292-7.

17. Sugita $\mathrm{H}$, Oda E, Hirota M, Ishikawa S, Tomiyasu S, Baba H, et al. Significance of lymphadenectomy with splenectomy in radical surgery for advanced (pT3/pT4) remnant gastric cancer. Surgery. 2016;159:1082-9.

Publisher's Note Springer Nature remains neutral with regard to jurisdictional claims in published maps and institutional affiliations. 